\title{
COMMENTARY
}

\section{Critical care support of patients with nicotine addiction}

\author{
Bekele Afessa* and Mark T Keegan \\ See related research by Lucidarme et al., http://ccforum.com/content/14/2/R58
}

\begin{abstract}
Over 500 million of the current world population will die from diseases caused by smoking cigarettes. The symptoms and signs of nicotine withdrawal are not well described in the critically ill. Since the various conditions of critical illness may lead to clinical manifestations mimicking nicotine withdrawal, describing its specific manifestations may not be easy. A few case reports suggest that nicotine replacement therapy may ameliorate nicotine withdrawal in the critically ill. However, retrospective studies have found that it may increase mortality. Despite the abundance of active smokers, there is a paucity of data describing nicotine withdrawal, and its prevention and treatment options in the critically ill. Future studies are warranted to address these issues.
\end{abstract}

In the previous issue of Critical Care, Lucidarme and colleagues [1] describe the impact of nicotine abstinence on the clinical course of critically ill patients receiving mechanical ventilation for at least 48 hours. Their study included 144 patients, 44 smokers and 100 non-smokers. The smokers were more likely to be younger and male, have a history of alcoholism, and be admitted for septic shock compared to non-smokers. The results of the study showed active smoking history to be an independent risk factor for agitation.

Lucidarme and colleagues address an important clinical issue intensivists face daily in our practice. There are 1.2 billion smokers in the world, half of whom will die from diseases caused by smoking [2]. Tobacco products are highly addictive $[3,4]$ and abstinence from their use in active smokers can lead to withdrawal symptoms $[5,6]$. These symptoms are often non-life threatening and are

*Correspondence: afessa.bekele@mayo.edu

Division of Pulmonary and Critical Care Medicine, Department of Internal Medicine, Mayo Clinic College of Medicine, Rochester, MN 55905, USA not well described in the critically ill. Mayer and collaborators [7] reported five cases of agitated delirium in smokers hospitalized for brain injury. They attributed the delirium to nicotine withdrawal because of its improvement following nicotine replacement therapy (NRT). Tran-Van and colleagues [8] reported a case with difficulty to wean from mechanical ventilation, and restlessness attributed to nicotine withdrawal. The patient's condition improved following NRT.

Because of several confounding factors, it is difficult to determine the exact incidence of nicotine withdrawal in critically ill smokers. Critical illness, mechanical ventilation, and sepsis can be associated with various levels of encephalopathy. The symptoms and signs of the encephalopathy may mimic the manifestations of nicotine withdrawal. Moreover, smokers may also have other addictions, including alcohol and illicit drugs. The manifestations of abstinence from such addictive substances may not be easily distinguishable from the manifestations of nicotine withdrawal. Although compromised by the small sample size, Lucidarme and colleagues [1] have done their best to minimize the impact of confounding factors by matching cases and controls.

There is a scarcity of data addressing the presence and extent of clinically important nicotine withdrawal symptoms in critically ill active smokers. The study by Lucidarme and colleagues is one step towards this. Since the symptoms and signs of nicotine withdrawal lack specificity in the critically ill, effective therapy aimed specifically at preventing and treating nicotine withdrawal, with no effect on other conditions with similar manifestations, may help to define the clinical course of nicotine withdrawal in the critically ill. The major currently available treatment options for nicotine addiction include NRT, bupropion, and varenicline. Several studies performed mostly in the outpatient setting have shown that NRT is safe and effective in ameliorating symptoms of nicotine withdrawal [9]. Although data justifying the use of NRT in the critically ill are nonexistent, some ICUs have developed protocols offering it to all active smokers [10]. We recognize that hospitalization provides an excellent opportunity to intervene on 
nicotine addiction; however, NRT may not be a harmless intervention. Although the study was compromised by several limitations, our groups have shown that NRT may be associated with increased mortality in the critically ill [10]. A similar association was reported in patients undergoing cardiac surgery [11]. We have recently completed a prospective, cohort study of active smokers admitted to a medical ICU [12]. The study did not show statistically significant association between NRT and mortality. However, NRT did not reduce the development of delirium.

Since nicotine addiction is a global health problem, some healthcare providers treat critically ill active smokers with NRT. Theoretically, this approach may be beneficial to critically ill smokers whose clinical course may be complicated by nicotine withdrawal. However, the clinical impact of nicotine withdrawal in the critically ill has not been well described. Although there are case reports suggesting that prevention and treatment of nicotine withdrawal may be beneficial $[7,8]$, retrospective studies have shown nicotine replacement therapy may increase mortality in the critically ill $[10,11]$. Future studies are warranted to describe the incidence and clinical manifestations of nicotine withdrawal. Moreover, interventions aimed at the prevention and treatment of nicotine withdrawal in the critically ill should be subjected to clinical trials before applying them to patients.

\section{Abbreviations}

NRT = nicotine replacement therapy

\section{Competing interests}

The authors declare that they have no competing interests.

\section{Authors' contributions}

Both authors wrote and approved the commentary.
Published: 17 May 2010

\section{References}

1. Lucidarme O, Seguin A, Daubin C, Ramakers M, Terzi N, Beck P, Charbonneau $\mathrm{P}, \mathrm{u}$ Cheyron D: Nicotine withdrawal and agitation in mechanically ventilated critically ill patients. Crit Care 2010, 14:R58.

2. Mackay JEM, Shafey O: The Tobacco Atlas. 2nd edition. Atlanta: American Cancer Society; 2006.

3. Hurt RD, Robertson CR: Prying open the door to the tobacco industry's secrets about nicotine: the Minnesota Tobacco Trial. JAMA 1998, 280:1173-1181.

4. Henningfield JE, Miyasato K, Jasinski DR: Abuse liability and pharmacodynamic characteristics of intravenous and inhaled nicotine. J Pharmacol Exp Ther 1985, 234:1-12.

5. Weinberger AH, Desai RA, McKee SA: Nicotine withdrawal in U.S. smokers with current mood, anxiety, alcohol use, and substance use disorders. Drug Alcohol Depend 2010, 108:7-12.

6. Van Zundert RM, Boogerd EA, Vermulst AA, Engels RC: Nicotine withdrawal symptoms following a quit attempt: an ecological momentary assessment study among adolescents. Nicotine Tob Res 2009, 11:722-729.

7. Mayer SA, Chong JY, Ridgway E, Min KC, Commichau C, Bernardini GL: Delirium from nicotine withdrawal in neuro-ICU patients. Neurology 2001 57:551-553.

8. Tran-Van D, Herve Y, Labadie P, Deroudilhe G, Avargues P: [Restlessness in intensive care unit: think to the nicotinic withdrawal syndrome]. Ann Fr Anesth Reanim 2004, 23:604-606.

9. Silagy C, Lancaster T, Stead L, Mant D, Fowler G: Nicotine replacement therapy for smoking cessation. Cochrane Database Syst Rev 2004:CD000146.

10. Lee $A H$, Afessa $B$ : The association of nicotine replacement therapy with mortality in a medical intensive care unit. Crit Care Med 2007, 35:1517-1521.

11. Paciullo CA, Short MR, Steinke DT, Jennings HR: Impact of nicotine replacement therapy on postoperative mortality following coronary artery bypass graft surgery. Ann Pharmacother 2009, 43:1197-1202.

12. Cartin-Ceba R, Afessa B: Nicotine replacement therapy in critically ill patients: A prospective observational cohort study [abstract]. Crit Care Med 2009, 37:A8 doi:10.1186/cc8955

Cite this article as: Afessa B, Keegan MT: Critical care support of patients with nicotine addiction. Critical Care 2010, 14:155. 